\title{
High-energy physics and cosmological perturbations: observing new physics at large scales.
}

\section{Diego CHIALVA ${ }^{* \dagger}$}

Université de Mons, Mons, Belgium

E-mail: diego.chialva@umons.ac.be

\begin{abstract}
Correlators of primordial perturbations could provide us with the signatures of physics at earlier times/higher momentum scales than inflation. The key-mechanisms are the interference and cumulation in time related to the interplay of negative- and positive-frequency components of the energy density generated by the high-momentum scale physics. Here, I discuss which signatures are universal for such scenarios, and which ones instead would distinguish the specific cases (for example modified initial states for inflationary perturbations or modified dispersion relations). I also discuss the scale dependence of the correlators in presence of these signatures, especially for some scenarios, and how it could be interesting for observations.
\end{abstract}

The European Physical Society Conference on High Energy Physics -EPS-HEP2013

18-24 July 2013

Stockholm, Sweden

\footnotetext{
* Speaker.

${ }^{\dagger}$ The work of of DC is supported by the Belgian National "Fond de la Recherche Scientifique" F.R.S.-F.N.R.S. with a contract "chargé de recherche".
} 


\section{Introduction}

One of the main successes of the inflationary paradigm is the prediction of "seeds" for structure formation, represented by (quantum) fluctuations of the fields during inflation. Several observations, present and soon-to-be, are sensitive to the these fluctuations already at the perturbative level, opening a window of opportunity to investigate the physics during inflation. However, as I will discuss in this short communication, cosmological observables can be used as a diagnostic of the physics at even higher momentum scales/ earlier times.

Research into these topics is of great importance nowadays, and not only for cosmology. Indeed, cosmological phenomena could be the only reliable way to investigate very high-energy physics, providing the arena to test theories and connect them with observations. This is even more significant now that the LHC has confirmed the missing elements of the Standard Model of particle physics, but not given hints so far of new physics, while astrophysical and cosmological observations present many challenges to our understanding.

In recent times there has been a large body of works on the topic of high-energy signatures in inflationary perturbation theory [1-7]. In this brief communication I will mainly deal with the general results after $[1,2]$, and discuss universality and specificity of the possible signatures.

\section{Cosmological observations and quantum theory of perturbations.}

Cosmological observations agree well with a description of the early universe in terms of a homogeneous isotropic background with a Friedman-Robertson-Walker metric (in conformal time)

$$
d s^{2}=a(\eta)^{2}\left(-d \eta^{2}+d \vec{x}^{2}\right),
$$

and an inflationary sector driving an early accelerated expansion, plus small perturbations. The latter ones are the seeds for structure formation and are strongly constrained by observations (initial energy density contrast of only $\frac{\delta \rho}{\rho} \sim 10^{-5}$ ).

Observations and consistency of the theory require a quantum description of the perturbations. In fact, due to the strong redshift, classical perturbations would need an initial energy density larger than the background one, thus invalidating the perturbative approach well supported by data. In the quantum theory this issue becomes just an indication that the initial state of the perturbations is the vacuum or nearly so ${ }^{1}$. Moreover, the inflationary redshift of physical scales is so strong that the perturbations that we observe today had a wavelength during inflation smaller than the Planck scale (transPlanckian initial conditions [7]): this should require a quantum treatment. Finally, the quantum theory explains the origin of the seeds themselves, as quantum fluctuations always appear due to the uncertainty principle and do not need to be postulated.

The cosmological observables are gauge invariant expectation values of operators in some initial state $\left|\Omega_{\text {in }}\right\rangle$. Using the interaction picture formalism:

$$
\left\langle\Omega_{\text {in }}|\widehat{\mathscr{O}}(\eta)| \Omega_{\text {in }}\right\rangle=\left\langle\Omega_{\text {in }}\left|\bar{T}\left(e^{i \int_{\eta_{\text {in }}}^{\eta} d \eta \widehat{\mathscr{H}}}\right) \widehat{\mathscr{O}}_{I}(\eta) T\left(e^{-i \int_{\eta_{\text {in }}}^{\eta} d \eta \widehat{\mathscr{H}}}\right)\right| \Omega_{\text {in }}\right\rangle,
$$

\footnotetext{
${ }^{1}$ Actually, quantum fluctuations have a non-zero vacuum energy density, which is in fact related to the cosmological constant issue. The usual attitude adopted when discussing inflationary perturbations is to ignore this zero-point contribution, even though this is questionable in principle.
} 
where $(\bar{T}) T$ is (anti)time-ordering, and, given the quadratic Hamiltonian of perturbations $\widehat{\mathscr{H}_{0}}(\eta)$,

$$
i \partial_{\eta} \widehat{\mathscr{O}}_{I}(\eta)=\left[\widehat{\mathscr{O}}_{I}(\eta), \widehat{\mathscr{H}}_{0}(\eta)\right] \text {. }
$$

\section{High-energy effects in cosmological perturbations.}

As we see from (2.2), observables are revealing of the physics during inflation, in particular interactions, background potential and universe expansion (via $\widehat{\mathscr{H}}_{I}$ and $\widehat{\mathscr{H}}_{0}$ ). However, they are also good diagnostic of earlier/higher momentum scale physics.

There are two ways to study the effects of new physics. A top-down one where the highenergy theory is modeled and the signatures are derived, and a bottom-up one, which I will adopt, where the high-energy physics is parametrized and its effects studied in terms of the parameters. In particular I will focus on 1) the dependence of observables on the initial state $\left|\Omega_{\text {in }}\right\rangle, 2$ ) the effects of corrections to the kinetic terms at momentum scales higher than inflation. As we will see, the signatures of these features are not irremediably washed out by the redshift during inflation.

I will deal with purely adiabatic perturbations, which are well-supported by observations as possible leading seeds of structure. Remarkably, adiabatic perturbations are conserved when their physical wavelength exceeds the Hubble scale, inverse of the Hubble rate $H \equiv \frac{\partial_{t} a}{a}$ ( $\partial_{t}$ is the cosmic time derivative). I will focus on scalar perturbations described by the gauge invariant comoving curvature perturbation $\widehat{\mathscr{R}}(\vec{x}, \eta)$, see [8] and reference there for its precise definition, and consider observables (2.2) given by correlators of this field.

The solution to the field equation (2.3) for $\widehat{\mathscr{R}}$ (dropping the label " $I$ ") is of the form

$$
\widehat{\mathscr{R}}(\vec{x}, \eta)=\int \frac{d^{3} k}{(2 \pi)^{3}}\left[\frac{f_{k}(\eta)}{z} \hat{a}_{k}^{\dagger}+\frac{f_{k}^{*}(\eta)}{z} \hat{a}_{k}\right] .
$$

where (primes stand for conformal time derivatives and $\phi$ is the background inflaton)

$$
f_{\vec{k}}^{\prime \prime}(\eta)+\left(\omega(\vec{k}, \eta)^{2}-\frac{z^{\prime \prime}}{z}\right) f_{\vec{k}}(\eta)=0 \quad z \equiv \frac{a \partial_{t} \phi}{H} .
$$

The initial state $\left|\Omega_{\text {in }}\right\rangle$ in (2.3) and the form of the dispersion relation $\omega(\vec{k}, \eta)$ derived from $\mathscr{H}_{0}$ dictate the high-momentum form of the mode functions $f_{\vec{k}}$. The standard choices are the adiabatic (also called Bunch-Davies) vacuum and the Lorentzian dispersion relation, defined as

$$
\hat{a}_{k}(\eta \rightarrow-\infty)\left|\Omega_{B D}\right\rangle=0, \quad \omega(\vec{k}, \eta)^{2}=k^{2} .
$$

The choice of the adiabatic vacuum is motivated by its similarity with the Minkowski one at very small scales and by the demand for a certain behaviour of the two-point functions (no antipodal poles).

However, the choices (3.3) can be questioned [7]. In fact, pre-inflation physics might have generated an excited initial state for inflationary perturbations (still satisfying backreaction constraints). Furthermore, from an effective theory viewpoint the states definition should not refer to scales way beyond the cutoff of the theory, as it is instead when $\eta \rightarrow-\infty$.

Similarly, Lorentz invariance has been tested only up to certain scales. Moreover, there exist theoretical scenarios where that symmetry is broken at short scales (for example Hořava's theory). This motivates investigating high-energy modifications to the dispersion relations. [1-3,7]

I will now discuss general effects on observables from modifications parametrizing new physics. 


\section{Power spectrum}

Observations of the CMBR depict a nearly Gaussian statistics of perturbations. An important observable is then the power spectrum ${ }^{2}$ after horizon exit $(\eta \rightarrow 0)$

$$
P_{k}=\lim _{\eta \rightarrow 0}\left\langle\mathscr{R}_{k}(\eta) \mathscr{R}_{k}(\eta)\right\rangle
$$

Standard scenario (labelled $s$ ) (adiabatic vacuum, Lorentzian dispersion, see (3.3)). In a nearly de Sitter background, where $\varepsilon \equiv-\frac{\dot{H}}{H} \ll 1$, typical of slow-roll and chaotic models, one finds

$$
P_{S}(k) \underset{k \rightarrow 0}{\sim} \frac{H^{2}}{4 M_{\text {Planck }}^{2} \varepsilon k^{3}}\left(\frac{k}{a H}\right)^{1-n_{s}},
$$

with $n_{s} \sim 0.96$ to comply with CMBR observations. $M_{\text {Planck }}$ is the reduced Planck mass.

Modified initial state (labelled $m i s$ ). If at the initial time $\eta_{\text {in }}$ some new physics above a momentum scale $\Lambda \gg H$ sets different initial conditions than the adiabatic vacuum, the mode functions have the form

$$
f_{k}^{(\mathrm{mis})}(\eta)=\alpha^{\mathrm{mis}}\left(k, \Lambda, \eta_{\text {in }}\right) f_{k}^{(\mathrm{s})}(\eta)+\beta^{\mathrm{mis}}\left(k, \Lambda, \eta_{\text {in }}\right) f_{k}^{(\mathrm{s}) *}(\eta),
$$

in terms of those found in the standard scenario, here indicated as $f_{k}^{(\mathrm{s})}(\eta)$. This leads to [7]

$$
P_{\text {mis }}(k) \underset{k \rightarrow 0}{\sim} \frac{H^{2}}{4 M_{\text {Planck }}^{2} \varepsilon k^{3}}\left(\frac{k}{a H}\right)^{1-n_{s}}\left(1+2 \operatorname{Re}\left(\beta_{k}^{\text {mis }} e^{i \operatorname{Argg}\left(\alpha_{k}^{\text {mis }}\right)}\right)\right)
$$

Modified dispersion relations (labelled $m d r$ ). A dispersion relation with corrections at a momentum scale $\Lambda \gg H$ can be written in general as

$$
\omega(\eta, k)=k Q\left(\frac{H}{\Lambda} k \eta\right), \quad \text { with } \quad Q(x \rightarrow 0) \rightarrow 1 .
$$

It is found that the field correlators are quite different from the standard scenario only if the adiabatic condition $\frac{\omega^{\prime}}{\omega^{2}} \ll 1$ is violated at some early time [2,3]. Then, even choosing initially the adiabatic vacuum, the field dynamics lead to a spectrum (see [2] for the precise result for the mode functions)

$$
P_{\text {mdr }}(k) \underset{k \rightarrow 0}{\sim} \frac{H^{2}}{4 M_{\text {Planck }}^{2} \varepsilon k^{3}}\left(1+2 \operatorname{Re}\left(\beta_{k}^{\text {mdr }}\right)\right) .
$$

with $\beta_{k}^{\text {mdr }}$ depending on $k$ and the interval of time $\Delta$ where WKB is violated (constrained by backreaction). Expanding for small $\Delta$, in full generality $\beta_{k}^{\text {mdr }}$ is proportional to $\Delta$, with coefficient given by the parameter signalling the violation of adiabaticity $[2,7]$.

\subsection{Universal and specific signatures}

Both modified initial states and modified dispersion relations lead to a distinctive signature: a modulation of the spectrum. The bottom-up approach I have been using is not able to further constrain the aspect of the modulation. Detailed scenarios and models, with their specific $\beta$ coefficients, can be distinguished by the different pattern of modulation and their amplitudes.

From the point of view of the observer these signatures are interpreted as the creation of particles $^{3}$ with an average number density of quanta $N_{\text {part }} \sim \int_{k}\left|\beta_{k}\right|^{2}$ leading to a pattern of interference due to "positive" and "negative energy" modes.

\footnotetext{
${ }^{2}$ Some authors define the spectrum rescaling the two-point function in our definition by $\frac{k^{3}}{2 \pi^{2}}$.

${ }^{3}$ I recall that the "particle" concept is not well-defined on time-evolving backgrounds, and neither is energy in absence of a global timelike Killing vector, but it is still useful as it relates easily to observations.
} 


\section{Non-Gaussianity}

The term non-Gaussianity indicates non-zero three- and higher-point correlators. The leading perturbative one is the bispectrum $\left.B\left(\vec{k}_{1}, \vec{k}_{2}, \vec{k}_{3}\right) \equiv\left\langle\mathscr{R}\left(\vec{k}_{1}\right) \mathscr{R}\left(\vec{k}_{2}\right) \mathscr{R}\left(\vec{k}_{3}\right)\right\rangle\right|_{\eta \sim 0}$ traditionally parametrized in terms of an amplitude $f_{N L}$ and a shape function with the momentum dependence. I write it as

$$
B\left(\vec{k}_{1}, \vec{k}_{2}, \vec{k}_{3}, \eta\right) \equiv(2 \pi)^{3} \delta\left(\sum_{i} \vec{k}_{i}\right)\left(-\frac{3}{5} f_{N L}\right)\left(\frac{H^{2}}{4 \varepsilon M_{\text {Planck }}^{2}}\right)^{2} \frac{4 \sum_{i} k_{i}^{-2}}{k_{t} \prod_{j} 2 k_{j}} F\left(\vec{k}_{1}, \vec{k}_{2}, \vec{k}_{3}\right) ; \quad k_{t} \equiv \sum_{i} k_{i}
$$

Clearly the bispectrum depends on the specific cubic couplings in the theory. In the case of the Einstein-Hilbert theory the leading one in derivative expansion is $H_{I}^{(e h)}=-\int d^{3} x a^{3}\left(\frac{\dot{\phi}}{H}\right)^{4} \frac{H}{M_{\text {Planck }}^{2}} \mathscr{R}^{\prime 2} \vec{\partial}^{-2} \mathscr{R}^{\prime}$. I take it as an example in the following, and consider again a quasi de Sitter background.

Standard scenario. For single field slow roll/chaotic models one finds [9]

$$
f_{N L} \approx-\frac{\dot{H}}{H^{2}} \ll 1, \quad F_{\mathrm{S}}\left(k_{1}, k_{2}, k_{3}, \eta\right)_{\mathrm{BD}}=1
$$

Modified initial state at a time $\eta_{\text {in }} \cdot{ }^{4}$ The bispectrum has on oscillating shape function, peaked on "folded configurations" $k_{j}=\sum_{h \neq j} k_{h}$, where the leading correction in $\beta$-expansion reads [6]

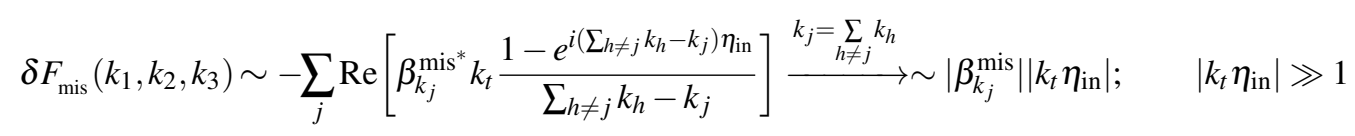

Modified dispersion relations. Obviously a Lorentz-violating theory presents new cubic couplings, but, more importantly, the modified mode functions lead to a shape function with a more complicated oscillating piece, and peaked on those momenta configurations for which there is an $n$ and a time $\eta_{*}$ such that $\partial_{\eta}^{m} \omega\left(k_{j}, \eta_{*}\right)=\sum_{h \neq j} \partial_{\eta}^{m} \omega\left(k_{h}, \eta_{*}\right) \forall m<n$ [2]. For these configurations

$$
\delta F_{\text {mdr }}\left(k_{1}, k_{2}, k_{3}\right) \rightarrow \sum_{j}\left(\frac{\Lambda}{H}\right)^{1-\frac{1}{n}} \frac{1}{\left|O_{k_{i}, \eta_{*}}\right|^{\frac{1}{n}}}\left|\beta_{\vec{k}_{j}}^{\text {mdr }^{*}}\right|,
$$

where $O_{k_{i}, \eta_{*}}$ is an order-one function fully specified in [2].

\subsection{Universal and specific signatures}

We can identify some generic types of signatures in the bispectrum from modified initial states and dispersion relations. Some are common to the two scenarios: an oscillating shape function, the possible presence of enhancements (depending on the smallness of $\beta$ ), and greater enhancements for interactions that scale with more powers of $\frac{1}{a}$, which means a stronger sensitivity to higher derivative couplings.

Other features distinguish the two cases: different patterns of oscillations and magnitude of "enhancements", and non-Gaussianities peaking for different configurations (in the case of modified dispersion relations they are strictly tied to the specific pattern of Lorentz violation [2]).

The physical interpretation of the signatures follows from three general points. In the standard scenario the largest contribution to non-Gaussianities is at late times ( $\sim$ horizon crossing), but in modified high-energy physics scenarios particle creation makes non-Gaussianities sizable also at early times. Furthermore, interference effects from "negative/positive-energy" components and $\mathrm{cu}$ mulative ones from time integration (consequence of time evolution) enhance these contributions.

\footnotetext{
${ }^{4}$ If different modes have different initial times $\eta_{\text {in }}^{k_{1,2,3}}$, the relevant one for equations (5.3), (5.4) is the latest one.
} 


\subsection{Squeezed limit and modified consistency relations}

Certain observations are sensitive to specific limits of the bispectrum $B\left(\vec{k}_{1}, \vec{k}_{2}, \vec{k}_{3}\right)$. For instance, the power spectrum of peaks in the matter distributions at large scale is related to the squeezed limit, where one of the wavenumber is much smaller than the others: say, $k_{1} \ll k_{2} \sim k_{3} \equiv k_{S}$.

Such limit is also relevant theoretically, because various consistency relations link the different correlators in such soft limits. The form of the relations distinguishes types of models. However, $[1,4]$ showed that the relations do not depend only on the couplings and on the background model, but also on the initial state of the perturbations and on their dispersion relations.

For example, for adiabatic perturbations one of these relations connects the bispectrum to the spectrum. In the standard scenario the relation is determined by powerful "generic" features: nonGaussianities peak at late times (horizon crossing), the "squeezed" $\mathscr{R}_{k_{1}}$ is superhorizon $\left(\left|k_{1} \eta\right| \ll 1\right)$ at peak time and acts as background for the other perturbations shifting their exit-of-horizon times. Thus, the limit is determined by the spectral index $n_{s}$ :

$$
\left\langle\mathscr{R}_{\vec{k}_{1}} \mathscr{R}_{\vec{k}_{2}} \mathscr{R}_{\vec{k}_{3}}\right\rangle_{\text {standard }} \underset{k_{1} \ll}{\sim}(2 \pi)^{3} \delta\left(\sum_{i} \vec{k}_{i}\right) \underbrace{\left(1-n_{s}\right)}_{O(\varepsilon)} \underbrace{P_{S}\left(k_{1}\right)}_{\sim k_{1}^{-3}} P_{S}\left(k_{S}\right)
$$

But in the cases of modified initial state (三mis) or modified dispersion relations ( $\equiv m d r$ ) the new physics leads to new general features [1]: 1) particle creation makes non-Gaussianities sizable already at earlier time $\eta_{n g} \sim-\frac{\Lambda}{H k_{s}}$, enhanced by interference and cumulation in time, 2) although $k_{1}$ is small (but non-zero), it can be both $\left|k_{1} \eta_{n g}\right| \gtrless 1$, so $\mathscr{R}_{k_{1}}$ may be subhorizon at the time of non-Gaussianities production. Therefore, the squeezed limit need not be fully determined by $n_{s}$.

For example, for the Einstein-Hilbert cubic coupling $H_{I}^{(e h)}$, see previous section, the standard result gets corrected by a term (leading in $\beta$ ) [1]

$$
\begin{aligned}
& \delta_{\beta}\left\langle\mathscr{R}_{\vec{k}_{1}} \mathscr{R}_{\vec{k}_{2}} \mathscr{R}_{\vec{k}_{3}}\right\rangle \underset{k_{1} \ll k_{S}}{\simeq}(2 \pi)^{3} \delta\left(\sum_{i} \vec{k}_{i}\right) 4 \varepsilon \mathscr{B} P_{S}\left(k_{1}\right) P_{S}\left(k_{S}\right),
\end{aligned}
$$

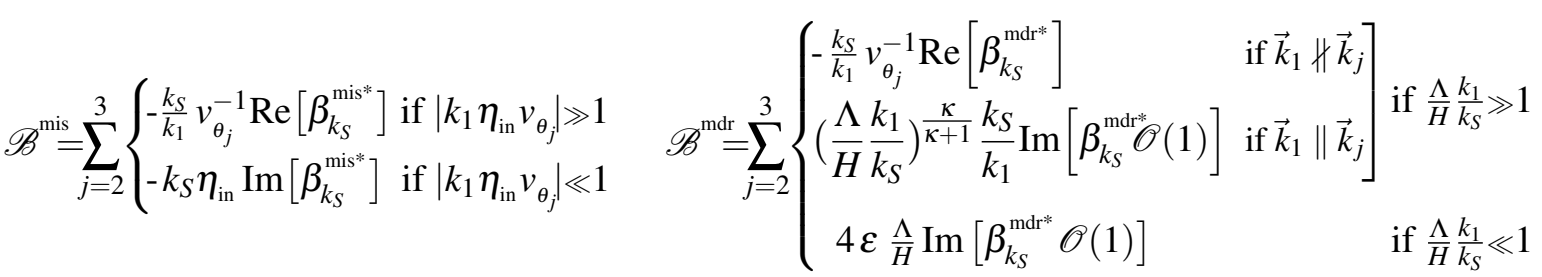

where $\kappa$ is the power of the leading correction in $\omega_{\text {phys }}(p) \sim p\left(1+c_{\kappa}\left(\frac{p}{\Lambda}\right)^{\kappa}+\ldots\right)$, and $v_{\theta_{j}}=(1+$ $\left.\cos \left(\theta_{j}\right)\right)$ with $\theta_{j}$ the angle between $\vec{k}_{1}$ and $\vec{k}_{j}$.

\subsection{Universal and distinctive features in the squeezed limit}

It is possible to make an analysis for all couplings using the effective theory for single-field inflation [1], to study universal and distinctive signatures in the squeezed limit, say, $k_{1} \ll k_{2,3} \sim k_{S}$.

In the standard scenario (adiabatic vacuum, Lorentz unbroken at all scales) the bispectrum is local $\left(\sim k_{1}^{-3}\right)$ in the squeezed limit, and has negligible amplitude $f_{N L} \sim 1-n_{s}=O(\varepsilon)$ determined by the spectral index.

With a modified initial state, the bispectrum has a non-local form $\left(\sim k_{1}^{-n}, n=4\right)$, if $\left|k_{1} \eta_{\text {in }} v_{\theta_{j}}\right| \gg 1$. In fact, one finds a local behaviour for a "new squeezed limit" $\left|k_{1} \eta_{\text {in }} v_{\theta_{j}}\right| \ll 1$ [1], but with a possibly enhanced amplitude (depending on the magnitude of $\left|\beta_{k_{S}}\right|$ ). 
With a modified dispersion relation, we also find a new, stronger squeezed limit. For $\frac{\Lambda}{H} \frac{k_{1}}{k_{S}} \ll 1$ the leading contribution is local and the amplitude enhanced (depending on the magnitude of $\beta$ ) as $f_{N L} \sim \varepsilon \frac{\Lambda}{H}\left|\beta_{k}\right|$, while higher derivative couplings, including Lorentz-breaking ones, are suppressed by additional powers of $\frac{k_{1}}{k_{S}}$. For $\frac{\Lambda}{H} \frac{k_{1}}{k_{S}} \gg 1$, the bispectrum is non-local, growing as $\sim k_{1}^{-4}$ for $k_{1} \ll k_{S}$. Higher-derivative couplings can grow as $k_{1}^{-n} n>4$, but are suppressed by $\left(\frac{H}{\Lambda}\right)^{m}, m \geq n$.

\section{Observations, comments and conclusions}

We have seen that primordial perturbations could provide us with a window on physics at a very high-momentum scale. The PLANCK collaboration has made a partial analysis [10] searching for indications of modified initial states at the level of spectrum and bispectrum, utilizing some templates. The results indicate that the templates for the modified states scenarios give good fit to the spectrum data (even better than the standard scenario), but the favoured part of the parameter space is fine tuned for the present sensitivity of the data. The analysis of non-Gaussianity (bispectrum) instead has not covered the whole (or large representative samples of) parameter space, but only four specific exemplary templates. The signal however has only a $1 \sigma$ significance.

What about future Planck and after Planck? The search for scale-dependent non-Gaussian signals hidden in the huge amount of data could benefit from the type of bispectrum with modified initial states or dispersion relations. In particular, modified dispersion relations lead to a scale dependence tied to the pattern of Lorentz violations. Resonances could occur for example for light massive fields. It is however necessary to reduce the parameter space, using a more top-down studies. Finally, LSS missions could tighten the constraints on these high-energy modifications.

\section{References}

[1] D. Chialva, JCAP 1210,037(2012).

[2] D. Chialva, JCAP 1201(2012)037 [arXiv:1106.0040 [hep-th]].

[3] A. Ashoorioon, D. Chialva and U. Danielsson, JCAP 1106(2011)034 [arXiv:1104.2338 [hep-th]].

[4] I. Agullo and L. Parker, Phys. Rev. D 83,063526(2011).

[5] J.O. Gong, M. Sasaki, Class. Quant. Grav. 30, 095005 (2013); R. Flauger, D. Green, R.A. Porto, arXiv:1303.1430 [hep-th]; S. Kundu, JCAP 1202,005(2012);

[6] X. Chen, M. Huang, S. Kachru, G. Shiu, JCAP 0701,002(2007). R. Holman, A. J. Tolley, JCAP 0805,001(2008). P. D. Meerburg, J. P. van der Schaar, P. S. Corasaniti, JCAP 0905,018(2009);

[7] J. Martin, R. H. Brandenberger, Phys. Rev. D 63,123501(2001); A. Kempf, Phys. Rev. D 63,083514(2001); R. Easther, B. R. Greene, W. H. Kinney, G. Shiu, Phys. Rev. D 64,103502(2001); N. Kaloper, M. Kleban, A. E. Lawrence, S. Shenker, Phys. Rev. D 66,123510(2002); U. H. Danielsson, Phys. Rev. D 66,023511(2002); K. Schalm, G. Shiu, J. P. van der Schaar, JHEP 0404,076(2004); D. Chialva, U. H. Danielsson, JCAP 0903(2009)007.

[8] Lyth, D. H., Riotto, A. 1999, Phys. Rep., 314, 1; Lidsey, J. E., Liddle, A. R., Kolb, E. W., et al. 1997, Reviews of Modern Physics, 69, 373.

[9] J. M. Maldacena, JHEP 0305,013(2003).

[10] P. A. R. Ade et al. [Planck Collaboration], arXiv:1303.5082 [astro-ph.CO]; arXiv:1303.5084 [astro-ph.CO]; arXiv:1303.5076 [astro-ph.CO]. 